\title{
Field-mediated self-assembly and actuation of highly parallel microfluidic devices
}

\author{
S. Bleila) \\ 2. Physikalisches Institut, Universität Stuttgart, Pfaffenwaldring 57, 70550 Stuttgart, Germany \\ D. W. M. Marr \\ Chemical Engineering Department, Colorado School of Mines, Golden, Colorado 80401 and \\ 2. Physikalisches Institut, Universität Stuttgart, Pfaffenwaldring 57, 70550 Stuttgart, Germany \\ C. Bechinger \\ 2. Physikalisches Institut, Universität Stuttgart, Pfaffenwaldring 57, 70550 Stuttgart, Germany
}

(Received 27 March 2006; accepted 12 May 2006; published online 29 June 2006)

\begin{abstract}
We present a macroscopic approach for the fabrication and actuation of microscale pumps in microfluidic environments. By applying a combination of rotating and static magnetic fields we overcome two fundamental issues preventing development of nanoscale systems: how does one assemble components in situ and how does one power these devices once in place? By using macroscopic fields we avoid the need for individual particle micromanipulation allowing for both scale down to the nanoscale and "scale up" to the simultaneous assembly and control of highly parallel device networks. (C) 2006 American Institute of Physics. [DOI: 10.1063/1.2217168]
\end{abstract}

Development of microfluidic devices for the control of small liquid flow or cell movement has recently received considerable attention because such devices are important and vital components of the complete laboratory on a chip. ${ }^{1}$ Also referred to as the micro total analysis system ( $\mu$ TAS) its eventual realization allows significant increase in density and complexity of microfluidic devices. Not only will highresolution micron-sized channel structures be required but also active components such as pumps and valves that can direct and control transport at these small length scales will be necessary. For the practical realization of microscale fluidic devices, two significant hurdles must be overcome. The first, fabrication, is currently being addressed through either improvements in the resolution of macroscale techniques ("top down") or through synthetic chemical approaches to create increasingly complex molecular structures ("bottom up,3). Though both approaches have achieved some success, the creation of working devices within their final functioning environments remains elusive. The second significant hurdle involves the delivery of energy across length scales to effectively power microdevices in a desired and controlled fashion once they have been created. ${ }^{4}$ This issue has limited the development of massively parallel arrays and complex microfluidic systems. In the past, approaches have been developed where colloidal particles have been employed and manipulated by optical tweezers. ${ }^{5}$ However, because each device must be created and powered individually, such techniques cannot be applied to assemblies with hundreds or even thousands of devices operating simultaneously.

Here we present a bulk-field-based in situ technique which allows the fabrication of micropumps from simple colloidal building blocks. In addition, the same field provides the power for operation of the pumps in their microenvironment once fabricated. By applying global fields we avoid the need for individual manipulation allowing for both scale down to the true nanoscale regime and the simultaneous as-

Konstanzer Online-Publikations-System (KOPS) URL: http://nbn-resolving.de/urn:nbn:de:bsz:352-0-41061

${ }^{a)}$ Electronic mail: s.bleil@ physik.uni-stuttgart.de sembly and control of highly parallel device networks.

Specifically, we employ external magnetic fields and superparamagnetic particles to create large numbers of microfluidic pumps. Such particles acquire a magnetic dipole moment when subjected to an external magnetic field. When the external field is applied in plane, the magnetic moments align leading to a mutual attraction of the particles forming compact particle clusters. ${ }^{6}$ If the field is applied normal to the microfluidic channels, the magnetic moments align perpendicular to the channels and repel one another. Because these fields can be easily extended over considerable lateral distances, fabrication of large arrays of colloidal microstructures is feasible. Channels were created with the use of soft lithography. ${ }^{7}$

Figures 1(a)-1(d) demonstrate the assembly of seven $4.5 \mu \mathrm{m}$ particles into a compact rotating cluster in the presence of a rotating magnetic field. ${ }^{8}$ Rotating magnetic fields in the colloidal plane were induced with three coils (diameter of $25 \mathrm{~mm}, 1000$ windings) positioned on a ring around the objective at a relative offset of $120^{\circ}$. Currents were applied to the coils using $100 \mathrm{~Hz}$ sine functions with a phase shift between neighboring coils of $120^{\circ}$. The maximum magnetic field was approximately $6 \mathrm{mT}$. Unlike other experiments where low field rotation frequencies lead to the formation of rotating chainlike structures, ${ }^{9}$ inside channels we observe that compact structures are always favored. This process also allows formation of independent clusters of different sizes, as shown in Figs. 1(e)-1(h). In this, three particles each form a particle cluster in the presence of a rotating field. An additional vertical field of about $3 \mathrm{mT}$ is then turned on, leading to a repulsion between clusters and the formation of two independent pumps. ${ }^{8}$ Note that cluster formation in these systems can be either reversible or irreversible depending on specific colloid surface chemistry and strength of the applied magnetic field.

Figures 2(a)-2(d) show a rotating cluster composed of seven particles in a microchannel structure filled with water. The external field rotates in a counterclockwise direction, inducing a torque on the cluster due to the interaction with 

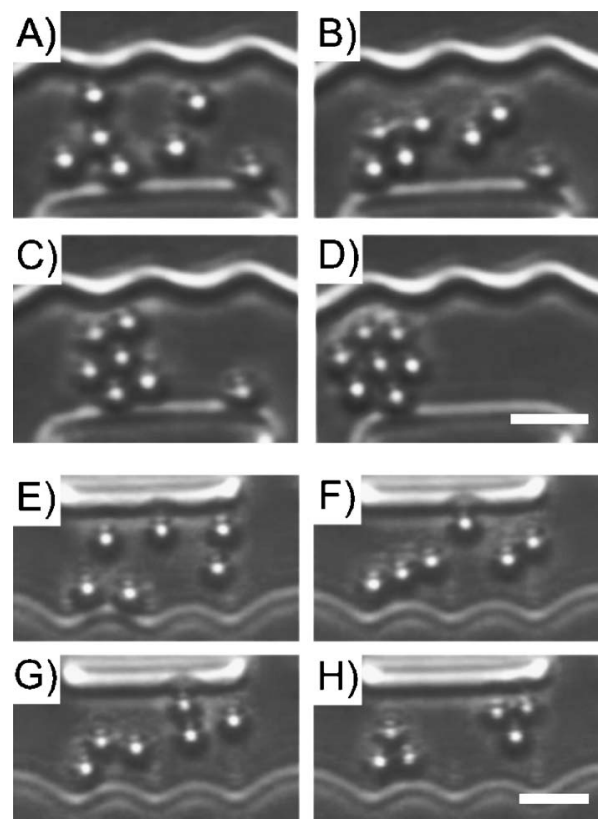

FIG. 1. Self-assembly of [(a)-(d)] seven $4.5 \mu \mathrm{m}$ magnetic particles in a channel structure into a compact micropump in the presence of an external magnetic field rotating in the colloidal plane. In this specific case, cluster formation is irreversible due to van der Waals forces and the pumps do not disassemble in the subsequent presence of repulsive particle interactions. (e)-(h). The size of the resulting micropumps can be adjusted by applying an additional static vertical magnetic field inducing a repulsive particle interaction. Here, after the formation of two three-particle clusters, the vertical field is turned on. In these studies, superparamagnetic colloidal particles (Dynabeads M-450 epoxy or M-270 carboxylic acid) of 4.5 or $2.7 \mu \mathrm{m} \mathrm{di-}$ ameter were employed. The experimental setup consists of a microscope, equipped with an optical tweezer (argon-krypton laser, $647 \mathrm{~nm}$ wavelength) guided by a telecentric lens system into the entrance aperture of the objective with a typical power of $20 \mathrm{~mW}$ in the sample cell. White bars correspond to $10 \mu \mathrm{m}$.

the magnetic dipoles. In the case here of a seven-particle cluster this leads to a maximum pump rotation frequency of approximately $20 \mathrm{~Hz}$ which is the result of the balance between viscous drag and the magnetic forces. The pump is situated in a structured channel with a maximal width of $16 \mu \mathrm{m}$ and height of $6 \mu \mathrm{m}$. In this, flow is visualized by the motion of nonmagnetic polystyrene tracer particles and measured by taking the time the tracer requires to travel through the bypass [indicated by the dotted line in Fig. 2(e)]. Measured for different field strengths, we find that, as expected, the pumping rate increases linearly with the strength of the rotating field [Fig. 2(f)].

Because of the laminar flow in microfluidic channels ${ }^{7}$ the solutions to the Navier-Stokes equations are time reversible and a rotating particle cluster can only induce a net flow if the channel symmetry is broken, as shown in Fig. 2(e). When applying a rotating field, the cluster tends to align itself close to the curved side of the channel. This becomes apparent when considering the flow created by the pump as seen when observing the tracer motion ${ }^{8}$ sketched in Fig. 3.

In our studies, the channels were designed to capture the pump in the asymmetric part and prevent translation along the wall because of the strong interaction between walls and cluster. This interaction can induce a small circular movement of the pump center of mass, which has no observable influence on the pump efficiency. In addition, however, the microchannel design plays an important role in device function. As these devices are powered using an external source,
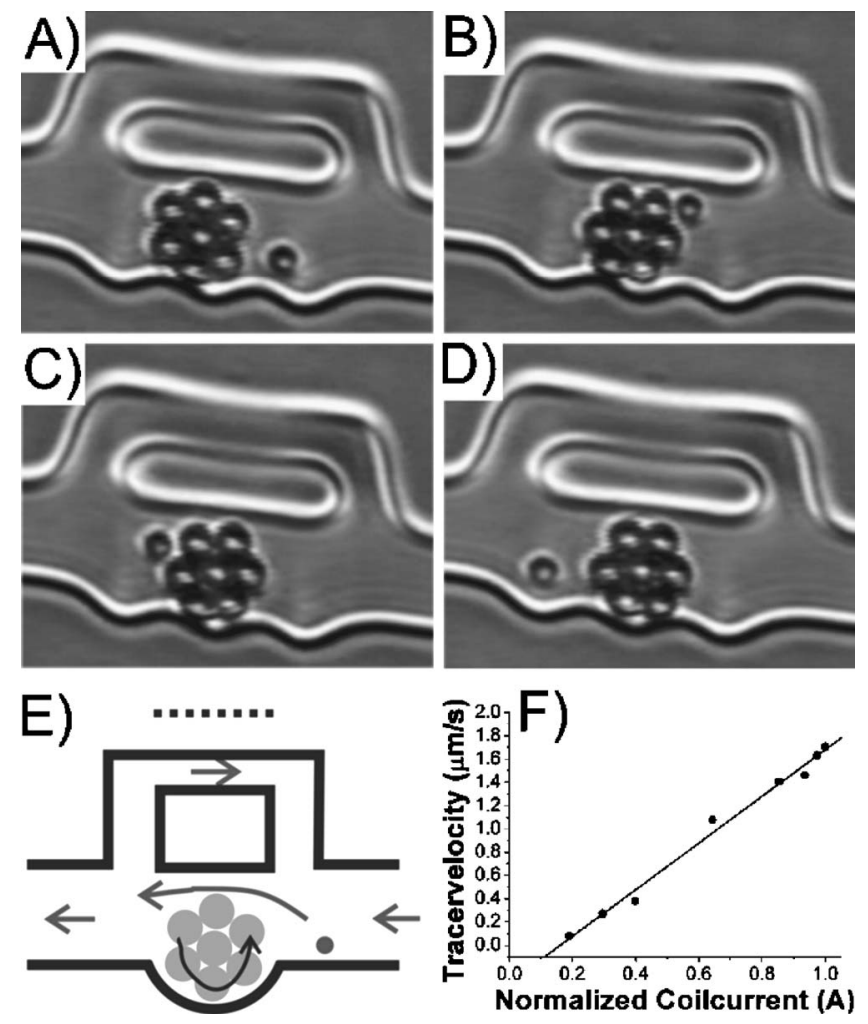

FIG. 2. Pumping mechanism. (a)-(d) Seven superparamagnetic particles (sterically stabilized with SDS) of $4.5 \mu \mathrm{m}$ diameter form a cluster driven counterclockwise by an external magnetic field that rotates at $100 \mathrm{~Hz}$ in the sample plane. The maximum width of the channels is $16 \mu \mathrm{m}$; the height is approximately $6 \mu \mathrm{m}$. Liquid flow is visualized with a $3 \mathrm{~m}$ polystyrene tracer particle (Ref. 8). (e) Illustration of the liquid flow arising from the rotating particle cluster. (f) Velocity of tracer particles as a function of the current through the magnetic coils. The linear fit is a guide for the eye. The flow rate is measured in the upper, approximately $5 \mu \mathrm{m}$ wide bypass channel.

their rotation is driven in the same direction, a feature that could limit function. However, pumping direction depends both on the cluster rotation direction and the channel geometry. As illustrated in Fig. 3 for pumps rotating in identical directions, net flow is determined by the location of the chan-

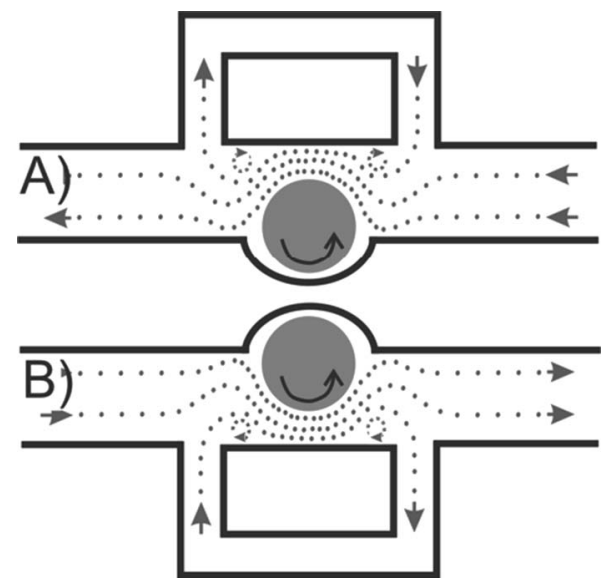

FIG. 3. Illustration of (a) the flow lines induced by the rotating cluster. Nearer points indicate faster liquid flow. The asymmetry in the pump geometry is necessary to create a net flow in one direction and for pump confinement where, on average, the cluster is localized in the asymmetry due to the Bernoulli effect. It is important to note that liquid flow can be reversed either by changing the cluster rotation direction or (b) by translating the cluster to a channel depression of opposite orientation. 


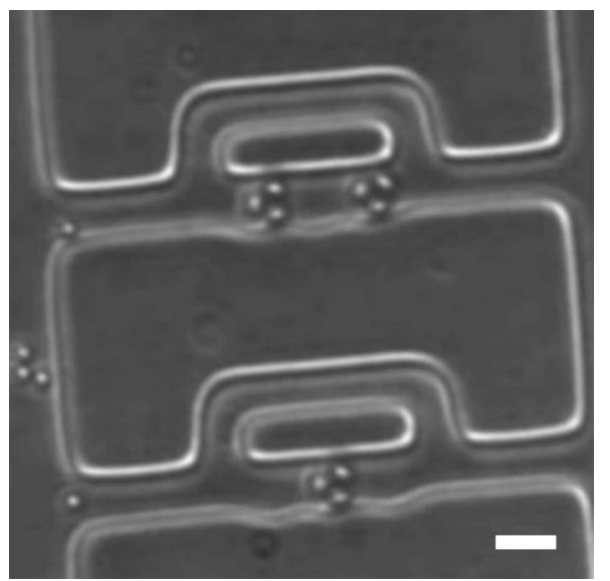

FIG. 4. Multiple colloidal pumps powered simultaneously. Because rotation rate is externally fixed, different pumping rates are achieved through the use of multiple colloidal assemblies. The white bar corresponds to $10 \mu \mathrm{m}$.

nel asymmetry. This illustrates that although pump assembly and powering are driven by the external field, pumping direction is determined by the physical geometry in which the pump is created.

In addition to pumps composed of seven particles, we have created pumps of two and three particles in similar geometries of varying channel width. We found that the pump efficiency increases with increasing pump diameter; more particles have a bigger collective magnetic moment, an effect that leads to faster rotation for a given applied magnetic field. These larger clusters also have more surface area leading to a stronger hydrodynamic interaction with the surrounding fluid. In addition, and due to the laminar nature of flows at the microscale, we find that pumps connected in series (Fig. 4) lead to greater flows than individual pumps.

The bulk-field approach presented here allows the simultaneous creation and actuation of significant numbers of micropumps inside microfluidic devices. This is demonstrated in Fig. 4 where each pump rotates in the same direction at approximately the same rate. Though denser configurations are easily possible, this image and an available movie ${ }^{8}$ of six pumps correspond to a pump density of approximately 30000 pumps $/ \mathrm{cm}^{2}$. Note that the energy required to drive all of these individual devices simultaneously is provided by a single external source.

For dynamic control of large pumping networks the ability to switch pumping direction within static channel designs is necessary. Here, one can take advantage of the interplay between local geometry and applied global field; local modi- fications to the magnetic field ${ }^{10}$ or application of a separate, supplementary field can alter local function. For example, as illustrated in Fig. 3, because the pumping direction depends on the pump position within the channel geometry, a simple translation of a micropump inside the channel structure will reverse flow if two neighboring channel concavities with opposite orientations are present. Because colloidal systems can react to a variety of fields, one could employ locally generated electric or magnetic fields to translate the colloidal clusters. However, because it is readily integrated within our experimental apparatus, we employed an optical tweezer ${ }^{11}$ to both translate the pump and reduce or even prevent the rotation of a single micropump by pressing it against the channel walls as demonstrated in Ref. 8.

We anticipate that this approach will be particularly useful for bioassaying and $\mu$ TAS applications in a variety of fields because these colloidal systems are well known for their biocompatibility and the ease with which biofunctionalities are incorporated on their surface. Not subject to diffraction limitations like previous approaches, the pumps presented here could be created at significantly smaller, nanoscopic length scales, increasing not only the density but also the complexity of the fluidic networks that could be fabricated.

${ }^{1}$ Proceedings of the Micro TAS 2001 Symposium (Kluwer Academic, Monterey, CA, 2001).

${ }^{2}$ S. Kawata, H. B. Sun, T. Tanaka, and K. Takada, Nature (London) 412, 697 (2001); H. G. Craighead, Science 290, 1532 (2000).

${ }^{3}$ A. H. Flood, J. F. Stoddart, D. W. Steuerman, and J. R. Heath, Science 306, 2055 (2004); J. D. Badjic, V. Balzani, A. Credi, S. Silvi, and J. F. Stoddart, ibid. 303, 1845 (2004).

${ }^{4}$ J. Leng, B. Lonetti, P. Tabeling, M. Joanicot, and A. Ajdari, Phys. Rev. Lett. 96, 084503 (2006); J. Goulpeau, D. Trouchet, A. Ajdari, and P. Tabeling, J. Appl. Phys. 98, 044914 (2005).

${ }^{5}$ D. G. Grier, Nature (London) 424, 810 (2003); A. Terray, J. Oakey, and D. W. M. Marr, Science 296, 1841 (2002).

${ }^{6}$ F. Amblard, B. Yurke, A. Pargellis, and S. Leibler, Rev. Sci. Instrum. 67, 818 (1996); L. Sacconi, G. Romano, R. Ballerini, M. Capitanio, M. De Pas, M. Giuntini, D. Dunlap, L. Finzi, and F. S. Pavone, Opt. Lett. 26, 1359 (2001).

${ }^{7}$ D. C. Duffy, J. C. McDonald, O. J. A. Schueller, and G. M. Whitesides, Anal. Chem. 70, 4974 (1998); G. M. Whitesides and A. D. Stroock, Phys. Today 54(6), 42 (2001).

${ }^{8}$ Compare movies at http://www.pi2.uni-stuttgart.de/Bechinger/research/ more/microfluidics/index.shtml

${ }^{9}$ S. Melle, O. G. Calderon, M. A. Rubio, and G. G. Fuller, Phys. Rev. E 68, 041503 (2003); Y. Naogaoka, H. Morimoto, and T. Maekawa, ibid. 71, 032502 (2005).

${ }^{10}$ T. Deng, G. M. Whitesides, M. Radhakrishnan, G. Zabow, and M. Prentiss, Appl. Phys. Lett. 78, 1775 (2001).

${ }^{11}$ K. C. Neuman and S. M. Block, Rev. Sci. Instrum. 75, 2787 (2004). 